\title{
Is there any superiority among diagnostic methods in organizing pneumonia in terms of clinical features of the patients?
}

This article was published in the following Dove Press journal:

Therapeutics and Clinical Risk Management

26 September 2016

Number of times this article has been viewed

\author{
Kuthan Kavakli' \\ Nesrin Ocal ${ }^{2}$ \\ Deniz Dogan ${ }^{2}$ \\ Ali Fuat Cicek $^{3}$ \\ Hakan Isik' \\ Sedat Gurkok' \\ 'Department of Thoracic Surgery, \\ ${ }^{2}$ Department of Chest Diseases, \\ ${ }^{3}$ Department of Pathology, \\ Gulhane Military Medical Academy, \\ Ankara, Turkey
}

Background: Organizing pneumonia (OP) can be idiopathic or secondary to some clinical situations. If an etiological cause is not present, this phenomenon is called cryptogenic OP. Secondary OP is associated with various diseases that are known to induce the OP.

Objectives: The aim of this study was to evaluate the clinical features of the cases with OP and compare the patients diagnosed by bronchoscopic transbronchial biopsy with patients diagnosed by surgical lung biopsy.

Patients and methods: Medical records of 41 patients diagnosed with OP between 2004 and 2014 were reviewed retrospectively.

Results: Totally, 41 patients with OP were identified. In all, $39.02 \%$ of the cases were diagnosed by bronchoscopic methods, and $60.97 \%$ of the cases were diagnosed by surgical procedures. Although the frequency of ground glass opacities, consolidations, and micronodules was higher in the group diagnosed by bronchoscopy, mass-like lesions were more common in the cases diagnosed by surgery. Bronchoscopy, performed in 30 patients totally, had a diagnostic efficacy of $53.33 \%$. Diagnostic value of bronchoscopy was significantly higher in cryptogenic OPs. Although diffuse radiological pattern was more common in "successful bronchoscopy" group, frequency of focal pattern was higher in "failed bronchoscopy" group. Ground glass opacity in successful bronchoscopy group and mass-like lesions in failed bronchoscopy group reached significant differences.

Conclusion: There were significant differences between the diagnostic procedures in terms of radiological patterns. This is the first study about the relationship between the diagnostic methods and the characteristics of OP.

Keywords: cryptogenic organizing pneumonia, secondary organizing pneumonia, clinical feature, diagnostic procedure

\section{Introduction}

Parenchymal organization is a common histopathological response to injury in the lung. While the organization usually regresses as a part of the normal healing process, repair may be self-reinforcing and leads to fibrosis in some cases. This clinical entity is defined as organizing pneumonia (OP), a distinct clinical entity. ${ }^{1}$ OP can be idiopathic or secondary to some clinical situations. If an etiological cause is not present, this phenomenon is called "cryptogenic OP (COP)", which is accepted as a subtype of idiopathic interstitial pneumonia. Secondary OP (SOP) is associated with various diseases that are known to induce the OP. ${ }^{2}$ It often occurs as a complication of existing chronic inflammatory diseases, including inflammatory bowel diseases, connective tissue diseases such as Sjögren's syndrome or rheumatoid arthritis,
Correspondence: Nesrin Ocal Department of Pulmonary Medicine and Intensive Care, Gulhane Medical School, 06010 Etlik, Ankara, Turkey

Tel +90505 5044715

Email nesrinbaygin@yahoo.com 
infection, malignancy, radiation treatment for lung or breast cancer, organ transplantation, and aspiration. OP may also be a side effect of certain medications such as amiodarone, methotrexate, nitrofurantoin, inhalation of toxic gases, cocaine, and human immunodeficiency virus infection. ${ }^{2-4}$ The histopathological pattern of OP is the formation of granulation tissue plugs within the small airways, alveolar ducts, and alveoli. ${ }^{3,4}$ Although the radiological findings including peripheral consolidation and bilateral airspace opacities are highly suggestive of OP and are not specific, the differential diagnosis comprises conditions such as the bronchoalveolar carcinoma, acute and chronic eosinophilic pneumonia, pulmonary lymphoma, pulmonary vasculitis, and sarcoidosis. ${ }^{5,6}$ The distinction between COP and SOP is important because the treatment and prognosis are different. While corticosteroids are the main treatment for COP, the management of SOP requires treatment of the underlying disease additionally. ${ }^{7,8}$ The distinction between COP and SOP can be made clinically, but there are limited data about this issue in the previous literature..$^{9-11}$ However, the possible advantages of diagnostic procedures to each other have also not been determined previously. In this retrospective study, we aimed to evaluate the clinical features of the COP and SOP cases and compare the patients diagnosed by bronchoscopic transbronchial biopsy (TBB) with patients diagnosed by surgical lung biopsy. This is the first study about the relationship between the diagnostic methods and the characteristics of COP and SOP cases.

\section{Patients and methods}

Medical records of 41 patients diagnosed with OP between January 1, 2004, and December 31, 2014, were reviewed retrospectively. The data including age, sex, symptoms, known etiological factors, thorax computed tomography (CT) findings, positron emission tomography/CT (PET/CT) characteristics, and maximum standardized uptake value (SUVmax) on F-fluoro-2-deoxy-D-glucose, pulmonary function test (PFT) results, diagnostic methods, and subtype of OP were recorded in all cases. The pathological slides were obtained from the archive of the pathology department, and they were reexamined by one attending pathologist, and the diagnosis of the cases was confirmed. Pathological samples were investigated blindly without any knowledge of radiological findings. Inflammatory reaction, intraalveolar accumulation of macrophages, fibrin exudation, and necrotic changes were considered as morphological but not specific criteria for OP. On the other hand, observation of Masson bodies (fibroblastic plugs filling the air spaces) was considered as a specific finding to establish a diagnosis of bronchiolitis obliterans organizing pneumonia (BOOP) pattern. After evaluating all of these morphological criteria, with a combination of radiological and clinical findings, an accurate diagnosis of OP or BOOP was made. Radiological images of all cases were evaluated by two pulmonologists, and consensus of their findings was recorded. They reviewed all the images, blinded to the clinical information of patients. The abnormalities in chest CT were classified as consolidation, ground glass opacity (GGO), mass-like lesion, nodule, cyst, micronodule, cavity, and reticular pattern. The PFT results, including forced vital capacity (FVC), percentage of forced vital capacity (FVC\%), forced expiratory volume in 1 second $\left(\mathrm{FEV}_{1}\right)$, percentage of forced expiratory volume in 1 second $\left(\mathrm{FEV}_{1} \%\right.$ ), and $\mathrm{FEV}_{1} / \mathrm{FVC}$ values, were interpreted as being normal, restrictive, obstructive, or mixed pattern, according to American Thoracic Society criteria. Diagnostic methods for all cases were determined, and patients were classified as diagnosed by bronchoscopic procedures and surgical procedures. Patients who underwent bronchoscopy were also divided into two groups according to the diagnostic results of the procedure; cases with diagnostic TBB results were included in successful bronchoscopy group, and cases with nondiagnostic TBB results were included in failed bronchoscopy group. All recorded data were statistically analyzed using SPSS 20.0 statistical software. $P$-value $<0.05$ was considered as statistically significant. Because the study was performed as a retrospective chart screening, informed consent was not obtained from the patients. The Medical Ethics Committee of Gulhane Military Medical Faculty (Ankara) approved the study protocol.

In descriptive statistics, frequency and percentage were used for discrete data, and mean $\pm \mathrm{SD}$ values were used for continuous variables. The normality of the continuous variables was analyzed with the Kolmogorov-Smirnov test and Shapiro-Wilks test. The Kruskal-Wallis and Mann-Whitney $U$ statistical tests were used for comparing the differences between the groups. Statistical significant results for $P$-values are bolded in the tables.

\section{Results \\ All OP patients}

Totally, 41 patients (27 men and 14 women) with a biopsyproven diagnosis of OP were identified. The mean age of the patients at presentation was $42.62 \pm 18.84$ years. Shortness of breath, cough, chest pain, and malaise were the most common symptoms in all OP cases (Table 1). The main radiological manifestations were GGO, consolidations, mass-like lesions, and cavities (Table 1; Figure 1A-D). PFT 
Table I Comparison of clinical, radiological, and PFT findings between COP and SOP cases

\begin{tabular}{|c|c|c|c|c|}
\hline & All OP $(n=4 I)$ & COP $(n=24)$ & SOP $(n=17)$ & $P$-value \\
\hline \multicolumn{5}{|l|}{ Symptom (\%) } \\
\hline Shortness of breath & 53.7 & 54.2 & 52.9 & 0.938 \\
\hline Cough & 43.9 & 41.7 & 47.1 & 0.732 \\
\hline Chest pain & 26.8 & 20.8 & 35.3 & 0.303 \\
\hline Weakness & 17.1 & 29.2 & 0 & 0.014 \\
\hline Hemoptysis & 9.8 & 8.3 & 11.8 & 1.000 \\
\hline Fever & 7.3 & 4.2 & 11.8 & 0.357 \\
\hline Asymptomatic & 9.8 & 12.5 & 5.9 & 0.482 \\
\hline \multicolumn{5}{|l|}{ Radiological findings (\%) } \\
\hline GGO & 43.9 & 54.2 & 29.4 & 0.116 \\
\hline Consolidation & 43.9 & 50 & 35.3 & 0.350 \\
\hline Mass-like lesion & 22 & 12.5 & 35.3 & 0.082 \\
\hline Nodule & 9.8 & 8.3 & 11.8 & 0.715 \\
\hline Reticular pattern & 4.9 & 8.3 & 0 & 0.222 \\
\hline Cyst & 9.8 & 16.7 & 0 & 0.076 \\
\hline Micronodule & 7.3 & 12.5 & 0 & 0.130 \\
\hline Cavity & 12.2 & 8.3 & 17.6 & 0.369 \\
\hline \multicolumn{5}{|l|}{$\mathrm{PFT}($ mean $\pm \mathrm{SD})$} \\
\hline $\mathrm{FVC}(\mathrm{L})$ & $3.62( \pm I .50)$ & $3.31( \pm \mathrm{I} .74)$ & $4.07( \pm 0.95)$ & 0.368 \\
\hline FVC (\%) & $104.2( \pm 64.5)$ & I I $2.9( \pm 83.0)$ & $91.4( \pm 13.6)$ & 0.904 \\
\hline $\mathrm{FEV}_{1}(\mathrm{~L})$ & $2.86( \pm 1.25)$ & $2.68( \pm \mathrm{I} .37)$ & $3.13( \pm 1.04)$ & 0.512 \\
\hline $\mathrm{FEV}_{1}(\%)$ & $95.4( \pm 67.7)$ & $102.6( \pm 86.2)$ & $84.9( \pm 23.9)$ & 0.753 \\
\hline $\mathrm{FEV}_{1} / \mathrm{FVC}$ & $78.3( \pm \mid 2.8)$ & $79.9( \pm 9.1)$ & $75.8( \pm 17.0)$ & 1.000 \\
\hline
\end{tabular}

Notes: Statistically significant results for $P$-values are shown in bold. The normality of the continuous variables was analyzed with the Kolmogorov-Smirnov test and ShapiroWilks test. The Kruskal-Wallis and Mann-Whitney $U$ statistical tests were used for comparing the differences between the groups. Statistically significant $P$-values $<0.05$ are printed in bold.

Abbreviations: PFT, pulmonary function test; COP, cryptogenic organizing pneumonia; SOP, secondary organizing pneumonia; OP, organizing pneumonia; GGO, ground glass opacity; FVC, forced vital capacity; FEV , forced expiratory volume in I second.
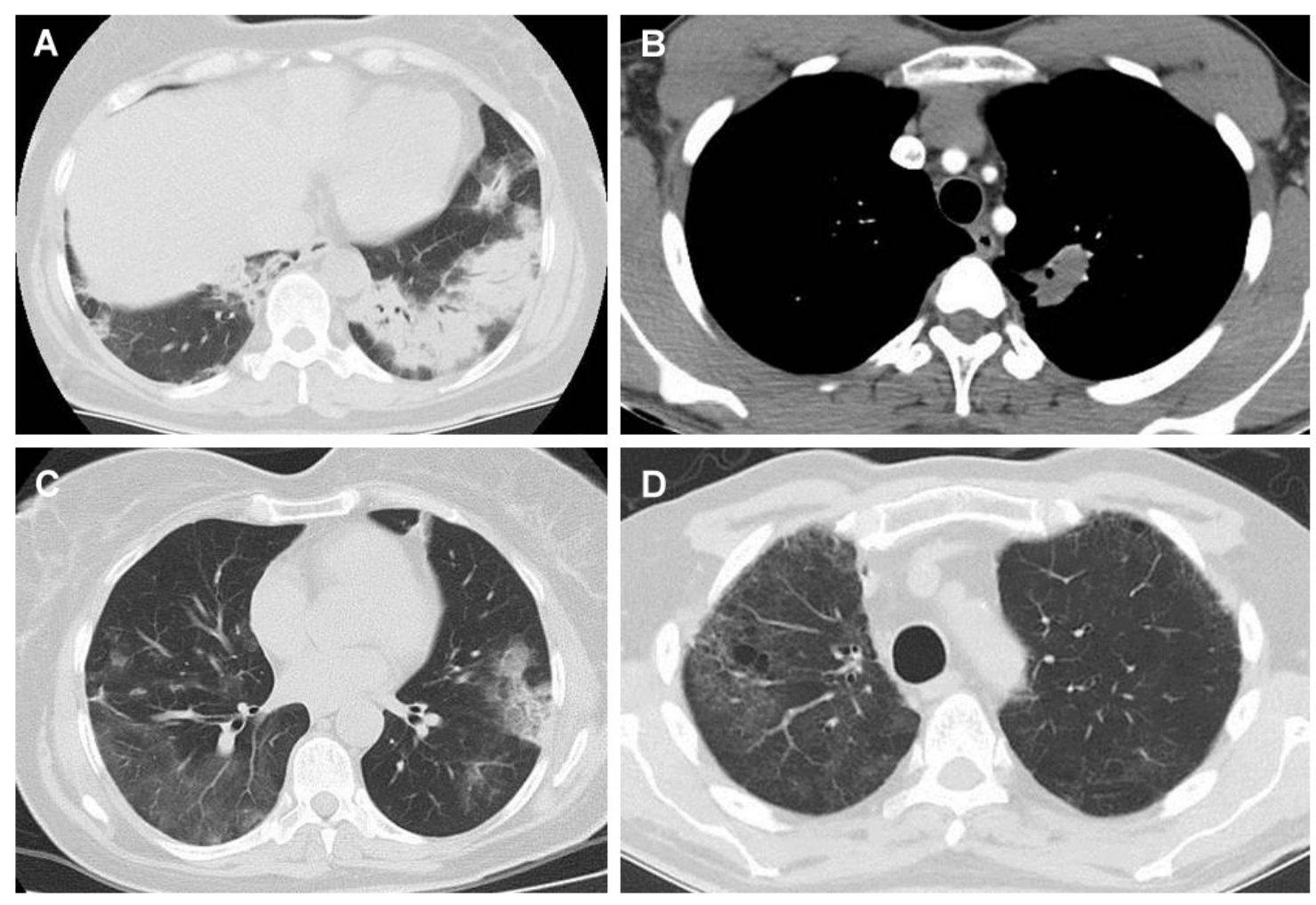

Figure I Thorax CT manifestations of organizing pneumonia cases. Consolidation (A), mass like lesion (B), ground glass opacity (C), cavity (D). Abbreviation: $\mathrm{CT}$, computed tomography. 
data were available for 27 patients. A reduced FVC was observed in $14.81 \%$ of patients, and a reduced $\mathrm{FEV}_{1}$ was observed in $33.33 \%$ of patients. While a total of $51.85 \%$ of the OP cases had normal PFT, 33.33\% had obstructive airflow pattern, and 14.81\% had mixed airflow pattern (Table 1). PET results that were available only in five cases were higher than normal limits with a mean SUVmax of 5.94. The diagnosis was made by surgical procedures in 25 (60.9\%) cases and by bronchoscopic methods in 16 (39.1\%) cases. Nonsurgical diagnostic procedures were performed in 15 cases (bronchoscopic methods in 14 cases and transthoracic needle aspiration in one case) before surgery but due to lack of an accurate diagnosis surgical procedures were performed (Figure 2A-C).

\section{SOP versus COP}

There were 24 (58.54\%; nine females and 15 males) COP patients and 17 (41.46\%; five females and 12 males) SOP patients. The mean ages of the patients at presentation were $44.38 \pm 20.08$ years in COP cases and $39.41 \pm 16.37$ years in SOP cases and did not show any significant difference. The underlying etiological causes in SOP patients were infectious pneumonia in six cases, acute myeloid leukemia in three cases, acute lymphocytic leukemia in one case, hydatid cyst in one case, breast carcinoma with hydatid cyst in one case, testicular carcinoma in one case, asthma with existence of rudimentary lung tissue in one case, and anti-nuclear antibody (ANA) with vasculitis in one case. Shortness of breath and cough were the first and second common symptoms in all cases. Malaise (in COPs) and chest pain (in SOPs) were the third common symptoms. Malaise was significantly more common in COPs ( $P=0.014$; Table 1$)$. While the main radiological manifestations were GGO and consolidations in COPs and mass-like lesions and GGO in SOP patients there was no statistically significant difference in radiological features among the two groups (Table 1). But there was no statistically significant difference in radiological features among the two groups (Table 1). PFT data were available for 16 COPs and eleven SOPs. Obstructive airflow pattern was identified in $36.36 \%$ of SOPs and $31.25 \%$ of COPs. Mixed airflow pattern was identified in $18.18 \%$ of SOPs and $12.5 \%$ of COPs. Mean FVC, mean $\mathrm{FEV}_{1}$, and mean $\mathrm{FEV}_{1} / \mathrm{FVC}$ values for SOPs were 4.07 L, 3.13 L, and 75.8, respectively, and that for COPs were $3.31 \mathrm{~L}, 2.68 \mathrm{~L}$, and 79.9, respectively (Table 1). PFT results did not reach significant difference between the two groups. While $50 \%(n=12)$ of COPs and $76.47 \%(n=13)$ of SOPs were diagnosed by surgical lung biopsy, $50 \%(n=12)$ of COPs and $23.53 \%(n=4)$ of SOPs were diagnosed by bronchoscopic biopsy.

\section{Bronchoscopy versus surgery}

Sixteen $(39.02 \%)$ cases were diagnosed by bronchoscopic methods and $25(60.97 \%)$ cases by surgical procedures (wedge biopsy, $n=17$; excisional biopsy, $n=4$; lobectomy, $\mathrm{n}=3$; and incisional biopsy, $\mathrm{n}=1$ ). Among cases diagnosed by surgical lung biopsy $(n=25), 48 \%(n=12)$ had COP and $52 \%(n=13)$ had SOP, whereas, among cases diagnosed by bronchoscopic biopsy $(n=16), 75 \%(n=12)$ had COP and $25 \%(n=4)$ had SOP. Shortness of breath was significantly more common in the group diagnosed by bronchoscopic methods ( $P=0.028$; Table 2 ). Although the frequency of GGO, consolidations, and micronodules in thorax CT were significantly higher in the group diagnosed by bronchoscopic methods ( $P=0.001, P=0.010$, and $P=0.025$, respectively), mass-like lesions were significantly more common in the cases diagnosed by surgical procedures $(P=0.007$; Table 2$)$. There was no significant difference in PFT results of these two groups (Table 2).

Taking into account the patients who underwent bronchoscopy previously to surgery $(n=14)$, bronchoscopic
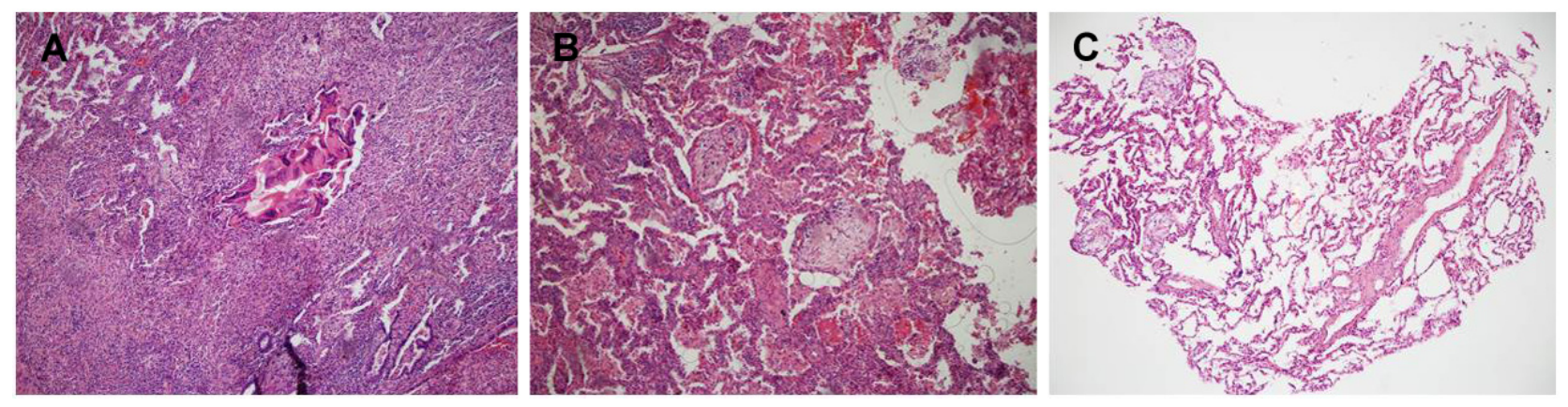

Figure 2 A foreign body tissue reaction against germinative membrane of probable hydatid cyst on histological examination of an excisional biopsy.

Notes: (A) There is a dense inflammatory reaction composed of lymphocytes, plasma cells, and histiocytes in the lung parenchyma surrounding the lesion (I00× H\&E). (B) In another area of the same slide, there are Masson bodies filling the airspaces (I00X H\&E). (C) Bronchoscopic biopsy sample containing many Masson bodies (I00× H\&E). 
Table 2 Comparison of clinical, radiological, and PFT findings between cases diagnosed by surgical lung biopsy and by bronchoscopic biopsy

\begin{tabular}{|c|c|c|c|c|}
\hline & All OP $(n=4 I)$ & $\begin{array}{l}\text { Bronchoscopic } \\
\text { diagnosis }(n=16)\end{array}$ & $\begin{array}{l}\text { Surgical } \\
\text { diagnosis }(n=25)\end{array}$ & $P$-value \\
\hline \multicolumn{5}{|l|}{ Symptom (\%) } \\
\hline Shortness of breath & 53.7 & 75.0 & 40.0 & 0.028 \\
\hline Cough & 43.9 & 56.2 & 36.0 & 0.202 \\
\hline Chest pain & 26.8 & 18.8 & 32.0 & 0.350 \\
\hline Weakness & 17.1 & 31.2 & 8.0 & 0.054 \\
\hline Hemoptysis & 9.8 & 0 & 16.0 & 0.092 \\
\hline Fever & 7.3 & 12.5 & 4.0 & 0.308 \\
\hline Asymptomatic & 2.4 & 6.2 & 0 & 0.206 \\
\hline \multicolumn{5}{|l|}{ Radiological findings (\%) } \\
\hline GGO & 43.9 & 75.0 & 24.0 & 0.001 \\
\hline Consolidation & 43.9 & 68.8 & 28.0 & 0.010 \\
\hline Mass-like lesion & 22.0 & 0 & 36.0 & 0.007 \\
\hline Nodule & 9.8 & 6.2 & 12.0 & 0.545 \\
\hline Reticular pattern & 4.9 & 6.2 & 4.0 & 0.744 \\
\hline Cyst & 9.8 & 18.8 & 4.0 & 0.120 \\
\hline Micronodule & 7.3 & 18.8 & 0 & 0.025 \\
\hline Cavity & 12.2 & 0 & 20.0 & 0.056 \\
\hline \multicolumn{5}{|l|}{$\mathrm{PFT}($ mean $\pm \mathrm{SD})$} \\
\hline $\mathrm{FVC}(\mathrm{L})$ & $3.62( \pm 1.50)$ & $3.49( \pm 1.16)$ & $3.70( \pm 1.69)$ & 0.359 \\
\hline FVC (\%) & $104.2( \pm 64.5)$ & $98.4( \pm \mid 2.4)$ & $107.5( \pm 8 \mid .5)$ & 0.243 \\
\hline $\mathrm{FEV}_{1}(\mathrm{~L})$ & $2.86( \pm 1.25)$ & $2.89( \pm 0.82)$ & $2.85( \pm 1.47)$ & 0.749 \\
\hline $\mathrm{FEV}_{1}(\%)$ & $95.4( \pm 67.7)$ & $86.3( \pm 27.3)$ & $100.7( \pm 83.4)$ & 0.749 \\
\hline $\mathrm{FEV}_{1} / \mathrm{FVC}$ & $78.3( \pm 12.8)$ & $81.9( \pm 9.87)$ & $76.1( \pm 14.1)$ & 0.473 \\
\hline
\end{tabular}

Notes: Statistically significant results for $P$-values are shown in bold. The normality of the continuous variables was analyzed with the Kolmogorov-Smirnov test and ShapiroWilks test. The Kruskal-Wallis and Mann-Whitney $U$ statistical tests were used for comparing the differences between the groups. Statistically significant $P$-values $<0.05$ are printed in bold.

Abbreviations: PFT, pulmonary function test; OP, organizing pneumonia; GGO, ground glass opacity; FVC, forced vital capacity; FEV in I second.

biopsies were performed in 30 patients totally. Considering that bronchoscopy was the main diagnostic method in 16 cases (successful bronchoscopy group) and it failed in 14 patients (failed bronchoscopy group), the general diagnostic efficacy of bronchoscopy was $53.33 \%$ in OP. Among the failed bronchoscopy group, there were nine SOP and five COP patients. Diagnostic value of bronchoscopy was significantly higher in COPs and significantly lower in SOPs $(P=0.030)$. While diffuse radiological pattern was significantly more common in successful bronchoscopy group, frequency of focal pattern was significantly higher in failed bronchoscopy group $(P=0.024)$. Additionally, GGO in successful bronchoscopy group and mass-like lesions in failed bronchoscopy group reached significant differences $(P=0.001$ in both; Table 3$)$.

\section{Discussion}

OP, previously defined as BOOP, is characterized by nonspecific symptoms such as flu-like illness. ${ }^{1,2,12}$ Malaise, cough, fever, and dyspnea are observed in more than two-thirds of

Table 3 Features of successful bronchoscopy group and failed bronchoscopy group

\begin{tabular}{|c|c|c|c|c|c|c|c|c|c|c|}
\hline & \multicolumn{3}{|c|}{$\begin{array}{l}\text { OP cases underwent } \\
\text { bronchoscopy }\end{array}$} & \multicolumn{3}{|c|}{ Radiological extent } & \multicolumn{4}{|c|}{ Radiological finding } \\
\hline & COP & SOP & $P$-value & Focal & Diffuse & $P$-value & GGO & $P$-value & Mass-like lesion & $P$-value \\
\hline \multicolumn{11}{|c|}{ Successful bronchoscopy group $(n=16)$} \\
\hline $\mathrm{n}$ & 12 & 4 & 0.030 & 7 & 9 & 0.024 & II & 0.001 & 0 & 0.001 \\
\hline$\%$ & 75 & 25 & & 43.8 & 56.2 & & 68.8 & & 0 & \\
\hline \multicolumn{11}{|c|}{ Failed bronchoscopy group $(n=14)$} \\
\hline $\mathrm{n}$ & 5 & 9 & & 13 & I & & 1 & & 7 & \\
\hline$\%$ & 35.7 & 64.3 & & 92.9 & 7.1 & & 7.1 & & 50 & \\
\hline
\end{tabular}

Notes: Statistically significant results for P-values are shown in bold. The normality of the continuous variables was analyzed with the Kolmogorov-Smirnov test and Shapiro-Wilks test. The Kruskal-Wallis and Mann-Whitney $U$ statistical tests were used for comparing the differences between the groups.

Abbreviations: OP, organizing pneumonia; COP, cryptogenic organizing pneumonia; SOP, secondary organizing pneumonia; GGO, ground glass opacity. 
the patients. ${ }^{3,13,14}$ Although hemoptysis and chest pain were previously described to be uncommon in many studies, we identified chest pain in $26.8 \%$ of our cases similar to the reports of Drakopanagiotakis et $\mathrm{al}^{11}$ and Lohr et al. ${ }^{15}$ On the other hand, fever (7.3\%) was significantly rare in our cases rather than available reports. ${ }^{2,12,16}$ Peripheral bilateral consolidations were previously reported to be the most common radiological findings in OP. ${ }^{9,10,17}$ In a partial accordance with previous reports, the main radiological manifestations were consolidation, GGO, mass-like lesion, and cavity in our cases. We observed that mass-like lesions (22\%) were more common in our patients rather than previous reports. ${ }^{3,11,18}$ Considering that pleural effusion is not observed in any of our cases, our results also confirmed the knowledge that pleural effusions are not common in OP..$^{12,14}$ Reversed halo sign (central GGOs surrounded by crescentic or ring-shaped consolidation), which is described as a unique finding for OP, was not present in our cases. ${ }^{19}$ Patients with OP usually present with a restrictive pattern on PFT but our results differed in this point with the previous literature. ${ }^{9,15}$ We observed normal PFT in $51.85 \%$, obstructive airflow pattern in $33.33 \%$, and mixed airflow pattern in $14.81 \%$ of the patients. Pure restrictive pattern was not observed in our cases. Consistent with the existing literature, PET results with a mean SUVmax level of 5.94 were at high levels that might interfere with malignancy. ${ }^{20}$

We found only three studies focused on the clinical and radiological distinctions of COPs and SOPs in the current English literature. ${ }^{9-11}$ In the study of Vasu et $\mathrm{al}^{9}$, fever and pleural effusion were reported to be significantly more common in SOPs, but there was no additional difference between COPs and SOPs. In the study of Sveinsson et $\mathrm{al}^{10}$, there was no major difference in the clinical features of COP and SOP, except that crackles were more common in SOPs. Drakopanagiotakis et $\mathrm{al}^{11}$ also reported that the clinical and radiographical findings in patients with $\mathrm{COP}$ and $\mathrm{SOP}$ were similar and nonspecific. In general, we did not identify significant differences between the clinical characteristics of patients with COP and SOP except for malaise, which was significantly more common in COPs. This situation was in accordance with previous studies. Although we identified that the main radiological manifestations were GGO and consolidations in COPs, and mass-like lesions and GGO in SOPs, we did not determine a statistically significant difference in radiological features among two groups similar to other studies. When we compared our results with other studies, we observed that the frequency of $\mathrm{CT}$ findings differed from previous reports. Consolidation had a rarer incidence in both groups in our study. In reverse to previous reports, we observed GGO more common in COPs and less common in SOPs..$^{10,11,21}$ Compared to former assessments, mass-like lesions were significantly more common in our SOP cases, and nodules were significantly less common in our COP cases. ${ }^{10,11,22}$

According to our knowledge, this is the first study focusing on the relationship between the diagnostic methods and the characteristics of COP and SOP cases with assessing the possible advantages of the diagnostic procedures to each other. Sveinsson et $\mathrm{al}^{23}$ researched the diagnostic methods for COPs and SOPs and reported that TBB was the main diagnostic method for both COPs and SOPs. They found that $76 \%$ of COP cases and $86 \%$ of SOP cases were diagnosed by TBB. Remaining cases were diagnosed by surgical lung biopsy, but there was no significant difference between the two groups. They did not evaluate the diagnostic efficacy of procedures and also their relationship with clinical and radiological features of COPs and SOPs. In our study, diagnostic value of surgical lung biopsy was higher than that of bronchoscopic biopsy. Diagnostic value of TBB was significantly higher in COPs rather than in SOPs $(P=0.030)$. On the other hand, radiological features were significantly different among diagnostic procedures. While the mass-like lesions were more common in cases diagnosed by surgical lung biopsy $(P=0.007)$, the frequency of GGO, consolidations, and micronodules was higher in the group diagnosed by bronchoscopic methods ( $P=0.001, P=0.010$, and $P=0.025$, respectively).

The relationship between the radiological findings and the diagnostic efficacy of bronchoscopy was demonstrated in various pulmonary diseases. ${ }^{24}$ However, we have not found any studies about this relationship in cases with OP. Our results show that radiological features were in association with the diagnostic efficacy of bronchoscopy. When the cases that underwent bronchoscopy were divided into two groups due to the diagnostic results of TBB as successful bronchoscopy and failed bronchoscopy groups, there were statistically significant differences in radiological findings. While diffuse radiological pattern was more common in successful bronchoscopy group, frequency of focal pattern was higher in failed bronchoscopy group $(P=0.024)$. Additionally, GGO in successful bronchoscopy group and mass-like lesions in failed bronchoscopy group reached significant differences ( $P=0.001$ in both). All these results may be interpreted as bronchoscopy tends to fail in cases with mass-like lesions and more sensitive in cases with GGO. Therefore, it can be suggested that clinicians should evaluate the patient together 
with radiological findings in the decision of the diagnostic procedure and should not insist on bronchoscopy in cases with mass-like lesions.

\section{Conclusion}

We did not identify any significant differences between the clinical and the radiological features of patients with COP and SOP except for malaise, which was significantly more common in COPs. On the other hand, we identified significant differences between the diagnostic procedures. Diagnostic value of TBB was significantly higher in COPs, and radiological features were in association with the diagnostic efficacy of the techniques. Considering that this is the first study about the relationship of diagnostic efficacy of surgical and bronchoscopic procedures with the clinical and radiological characteristics of OP cases, we hope that it would shed light on this issue. Being a retrospective study was the main limitation of our study. Further prospective studies are needed to support our results.

\section{Author contributions}

All authors contributed toward data analysis, drafting and critically revising the paper and agree to be accountable for all aspects of the work.

\section{Disclosure}

The authors report no conflicts of interest in this work.

\section{References}

1. Colby TV. Pathologic aspects of bronchiolitis obliterans organising pneumonia. Chest. 1992;102(1):38-43.

2. Dogan D, Ocal N, Yucel O, Tasci C, Gunal A. Cryptogenic organizing pneumonia diagnosed with transbronchial parenchymal biopsy: a case report with accompanying histopathological images. J Turgut Ozal Med Cent. 2016;23(1):81-83.

3. Lazor R, Vandevenne A, Pelletier A, et al. Cryptogenic organizing pneumonia. Characteristics of relapses in a series of 48 patients. The Groupe d'Etudes et de Recherche sur les Maladles "Orphelines" Pulmonaires (GERM“'O”P). Am J Respir Crit Care Med. 2000;162(2 Pt 1): 571-577.

4. Epler GR. Bronchiolitis obliterans organising pneumonia. Arch Intern Med. 2001;161(2):158-164.

5. Alsaghir AH, Al-Mobeireek AF, Al-Jahdali H, et al. Bronchiolitis obliterans organizing pneumonia: experience at three hospitals in Riyadh. Ann Saudi Med. 2007;27:32-35.
6. Ocal N, Dogan D, Ocal R, et al. Effects of radiological extent on neutrophil/ lymphocyte ratio in pulmonary sarcoidosis. Eur Rev Med Pharmacol Sci. 2016;20(4):709-714.

7. Basarakodu KR, Aronow WS, Nair CK, et al. Differences in treatment and in outcomes between idiopathic and secondary forms of organizing pneumonia. Am J Ther. 2007;14(5):422-426.

8. Kligerman SJ, Franks TJ, Galvin JR. From the radiologic pathology archives: organization and fibrosis as a response to lung injury in diffuse alveolar damage, organizing pneumonia, and acute fibrinous and organizing pneumonia. Radiographics. 2013;33(7):1951-1975.

9. Vasu TS, Cavallazzi R, Hirani A, Sharma D, Weibel SB, Kane GC. Clinical and radiologic distinctions between secondary bronchiolitis obliterans organizing pneumonia and cryptogenic organizing pneumonia. Respir Care. 2009;54(8):1028-1032.

10. Muller NL, Staples CA, Miller RR. Bronchiolitis obliterans organizing pneumonia: CT features in 14 patients. Am J Roentgenol. 1990;154: 983-987.

11. Drakopanagiotakis F, Paschalaki K, Abu-Hijleh M, et al. Cryptogenic and secondary organizing pneumonia: clinical presentation, radiographic findings, treatment response, and prognosis. Chest. 2011;139(4):893-900.

12. Oymak FS, Demirbaş HM, Mavili E, et al. Bronchiolitis obliterans organizing pneumonia. Clinical and roentgenological features in 26 cases. Respiration. 2005;72(3):254-262.

13. Oikonomou A, Hansell DM. Organizing pneumonia: the many morphological faces. Eur Radiol. 2002;12(6):1486-1496.

14. Chang J, Han J, Kim DW, et al. Bronchiolitis obliterans organizing pneumonia: clinicopathologic review of a series of 45 Korean patients including rapidly progressive form. J Korean Med Sci. 2002;17(2): $179-186$.

15. Lohr RH, Boland BJ, Douglas WW, et al. Organizing pneumonia. Features and prognosis of cryptogenic, secondary, and focal variants. Arch Intern Med. 1997;157(12):1323-1329.

16. Stove DE, Mangio D. Macrolides: a treatment alternative for bronchiolitis obliterans organising pneumonia? Chest. 2005;128(5):3611-3617.

17. Cordier JF. Organising pneumonia. Thorax. 2000;55(4):318-328.

18. Cazzato S, Zompatori M, Baruzzi G, et al. Bronchiolitis obliteransorganizing pneumonia: an Italian experience. Respir Med. 2000; 94(7):702-708.

19. Kim SJ, Lee KS, Ryu YH, et al. Reversed halo sign on high resolution CT of cryptogenic organizing pneumonia: diagnostic implications. AJR Am J Roentgenol. 2003;180(5):1251-1254.

20. Erdoğan Y, Özyürek BA, Özmen Ö, et al. The evaluation of FDG PET/CT scan findings in patients with organizing pneumonia mimicking lung cancer. Mol Imaging Radionucl Ther. 2015;24(2):60-65.

21. Drakopanagiotakis F, Polychronopoulos V, Judson MA. Organizing pneumonia. Am J Med Sci. 2008;335(1):34-39.

22. Epler GR. Bronchiolitis obliterans organizing pneumonia, 25 years: a variety of causes, but what are the treatment options? Expert Rev Respir Med. 2011;5(3):353-361.

23. Sveinsson OA, Isaksson HJ, Sigvaldason A, Yngvason F, Aspelund T, Gudmundsson G. Clinical features in secondary and cryptogenic organising pneumonia. Int J Tuberc Lung Dis. 2007;11(6):689-694.

24. Ocal N, Bilgic H, Tozkoparan E, et al. The relationship between diagnostic efficiency of bronchoscopy with clinical and radiological findings in pulmonary sarcoidosis. Turkiye Klinikleri Arch Lung. 2015; 16(2):31-39.
Therapeutics and Clinical Risk Management

\section{Publish your work in this journal}

Therapeutics and Clinical Risk Management is an international, peerreviewed journal of clinical therapeutics and risk management, focusing on concise rapid reporting of clinical studies in all therapeutic areas, outcomes, safety, and programs for the effective, safe, and sustained use of medicines. This journal is indexed on PubMed Central, CAS,

\section{Dovepress}

EMBase, Scopus and the Elsevier Bibliographic databases. The manuscript management system is completely online and includes a very quick and fair peer-review system, which is all easy to use. Visit http://www.dovepress.com/testimonials.php to read real quotes from published authors. 